\title{
ON THE RODMAN-SHALOM CONJECTURE REGARDING THE JORDAN FORM OF COMPLETIONS OF PARTIAL UPPER TRIANGULAR MATRICES*
}

\author{
CRISTINA JORDÁN ${ }^{\dagger}$, JUAN R. TORREGROSA ${ }^{\dagger}$, AND ANA M. URBANO ${ }^{\dagger}$
}

Dedicated to Hans Schneider on the occasion of his seventieth birthday.

Abstract. Rodman and Shalom [Linear Algebra and its Applications, 168:221-249, 1992] conjecture the following statement: Let $A$ be a lower irreducible partial upper triangular $n \times n$ matrix over $F$ such that $\operatorname{trace}(A)=0$. Let $n_{1} \geq n_{2} \geq \cdots \geq n_{p} \geq 1$ be a set of $p$ positive integers such that $\sum_{i=1}^{p} n_{i}=n$. There exists a nilpotent completion $A_{c}$ of $A$ whose Jordan form consists of $p$ blocks of sizes $n_{i} \times n_{i}, i=1,2, \ldots, p$ if and only if $r\left(A^{k}\right) \leq \sum_{i: n_{i} \geq k}\left(n_{i}-k\right), k=1,2, \ldots, n_{1}$.

In this paper this conjecture is solved in two cases: when the minimal rank of $A$ is 2 , and for matrices of size $5 \times 5$.

Key words. Completion problems, Jordan structure, Minimal rank, Majorization.

AMS subject classifications. 15A18, 15A21

1. Introduction. We consider $n \times n$ matrices $A=\left[a_{i j}\right]$ over an infinite field $\mathbb{F}$. A matrix is said to be a partial matrix if some of its entries are given elements from the field $\mathbb{F}$, while the rest of them can be arbitrarily chosen and treated as free independent variables. If those last elements are fixed, the resultant matrix is called a completion $A_{c}$ of the matrix $A$. The completion problems consist of finding all the completions of a given partial matrix with prescribed properties. We are interested in partial upper triangular matrices, namely, partial matrices $A=\left[a_{i j}\right]$ where $a_{i j}, i \leq j$, are the given elements. For this kind of matrices some completion problems have been studied. For example, problems related to the rank of the matrix can be found in [9], to eigenvalues in $[1,9]$, to Jordan form in $[2,7,8,9]$ and to controllability of linear systems in $[3,4]$.

Let $A$ be a partial matrix. We denote by $r(A)$ the minimal rank of all possible completions of $A$, that is, $r(A)=\min _{A_{c}} \operatorname{rank}\left(A_{c}\right)$ where the minimum is taken over the set of all the ranks of possible completions of $A$. Moreover, for any positive integer $k$, we denote $r\left(A^{k}\right)=\min _{A_{c}} \operatorname{rank}\left(\left(A_{c}\right)^{k}\right)$, where the minimum is taken over the set of all the ranks of the $k$ th power of possible completions of $A$.

We recall that a matrix $A$ is lower similar to a matrix $B$ (we denote $A \sim B$ ) if there exists a lower triangular matrix $S$ such that $A=S B S^{-1}$. In addition, a matrix $A$ of size $n \times n$ is said to be lower irreducible if all its $k \times(n-k)$ submatrices

*Received by the editors on 10 September 1996. Accepted for publication on 16 June 1998. Handling Editor: Daniel Hershkowitz.

${ }^{\dagger}$ Departamento de Matemática Aplicada, Universidad Politécnica de Valencia, 46020 Valencia, Spain (cjordan@mat.upv.es, jrtorre@mat.upv.es, amurbano@mat.upv.es). Research supported by Spanish DGICYT grant number PB94-1365-C03-02 
$\left[a_{i j}\right]_{i=1, j=k+1}^{k,} \underset{n}{n}$ are nonzero for $k=1,2, \ldots, n-1$.

Given two nonincreasing sequences of nonnegative integers $\left\{k_{i}\right\}_{i=1}^{p}$ and $\left\{q_{i}\right\}_{i=1}^{r}$, we say that $\left\{k_{i}\right\}_{i=1}^{p}$ majorizes $\left\{q_{i}\right\}_{i=1}^{r}$, denoted $\left\{k_{i}\right\}_{i=1}^{p} \succ\left\{q_{i}\right\}_{i=1}^{r}$, if

$$
\sum_{i=1}^{s} k_{i} \geq \sum_{i=1}^{s} q_{i}, \quad s=1,2, \ldots, p, \quad \text { and } \quad \sum_{i=1}^{p} k_{i}=\sum_{i=1}^{r} q_{i} .
$$

Rodman and Shalom give in [9] the following completion problem,

CONJECTURE: Let $A$ be a lower irreducible partial upper triangular $n \times n$ matrix over $\mathbb{F}$ such that trace $(A)=0$. Let $n_{1} \geq n_{2} \geq \cdots \geq n_{p} \geq 1$ be a set of $p$ positive integers such that $\sum_{i=1}^{p} n_{i}=n$. There exists a nilpotent completion $A_{c}$ of $A$ whose Jordan form consists of $p$ blocks of sizes $n_{i} \times n_{i}, i=1,2, \ldots, p$ if and only if

$$
r\left(A^{k}\right) \leq \sum_{i: n_{i} \geq k}\left(n_{i}-k\right), \quad k=1,2, \ldots, n_{1} .
$$

Note that the right hand side of (1) is the rank of $A_{c}^{k}$ provided that $A_{c}$ is a nilpotent completion of $A$ with the described Jordan form. The necessity of (1) is therefore evident.

Rodman and Shalom prove the above conjecture when $r(A)=1$ (see [9], Theorem 3.2 ) or in general for matrices of size $n \leq 4$ (see [9], Theorem 3.3). In [5] we prove that this conjecture is not true in general for matrices with minimal rank equal to three and for matrices of size $n \times n, n \geq 6$.

In this paper we prove that this conjecture is true in two remaining cases: when $r(A)=2$, and for matrices of size $5 \times 5$.

Let $A_{0}$ be the completion obtained by replacing the unspecified elements by zero. We denote by $J_{q}(\lambda)$ the $q \times q$ Jordan block with eigenvalue $\lambda$. The block diagonal matrix with diagonal blocks $A_{1}, \ldots, A_{t}$ is denoted by $A_{1} \oplus \cdots \oplus A_{t}$. Finally, given a matrix $A=\left[a_{i j}\right]$ let $a_{i j}$ represent the corresponding nonzero entries of any similar matrix obtained.

2. The main result. We prove the conjecture of Rodman and Shalom for matrices with minimal rank equal to two.

THEOREM 2.1. Let $A$ be an $n \times n$ lower irreducible partial upper triangular matrix such that $\operatorname{trace}(A)=0$ and $r(A)=2$. Let $n_{1} \geq n_{2} \geq \cdots \geq n_{p} \geq 1$ be a set of $p$ positive integers such that $\sum_{i=1}^{p} n_{i}=n$. If

$$
r\left(A^{k}\right) \leq \sum_{i: n_{i} \geq k}\left(n_{i}-k\right), \quad k=1,2, \ldots, n_{1}
$$

then there exists a nilpotent completion $A_{c}$ of $A$ whose Jordan form consists of $p$ blocks of sizes $n_{i} \times n_{i}, i=1,2, \ldots, p$.

Proof. By lower similarity we can transform the matrix $A$ into a partial upper triangular matrix $A_{1}$ which has only two nonzero rows. Since $A$ is lower irreducible, 
one of this rows is the first one. We assume that the other one is the jth row. Then the matrix $A_{1}$ has the following structure:

$$
A_{1}=\left[\begin{array}{ccccccccc}
a_{11} & a_{12} & \cdots & a_{1 j-1} & a_{1 j} & a_{1 j+1} & \cdots & a_{1 n-1} & a_{1 n} \\
& 0 & \cdots & 0 & 0 & 0 & \cdots & 0 & 0 \\
& & \ddots & \vdots & \vdots & \vdots & & \vdots & \vdots \\
& & & 0 & 0 & 0 & \cdots & 0 & 0 \\
& & & & a_{j j} & a_{j j+1} & \cdots & a_{j n-1} & a_{j n} \\
& & & & & 0 & \cdots & 0 & 0 \\
& & & & & & \ddots & \vdots & \vdots \\
& & & & & & & 0 & 0
\end{array}\right] .
$$

Since the matrix $A_{1}$ is lower irreducible its nth column has a nonzero entry. We can distinguish the following cases:

(a) $a_{1 n} \neq 0$.

By lower similarity we cancel the $(j, n)$ th entry and all the entries in the first row, except the $(1,1)$ th entry. Since the matrix $A_{1}$ is lower similar to the matrix $A$, its minimal rank is equal to two, and then the jth row has a nonzero entry. Let us assume that:

(a1) $a_{j t}, j<t<n$, is the first nonzero entry beginning from the right.

By lower similarity we can cancel all the entries in this row, except the element $a_{j j}$. We obtain the matrix

$$
A_{2}=\left[\begin{array}{cccccccc}
a_{11} & 0 & \cdots & 0 & \cdots & 0 & \cdots & a_{1 n} \\
& 0 & \cdots & 0 & \cdots & 0 & \cdots & 0 \\
& & \ddots & \vdots & & \vdots & & \vdots \\
& & & a_{j j} & \cdots & a_{j t} & \cdots & 0 \\
& & & & \ddots & \vdots & & \vdots \\
& & & & & 0 & \cdots & 0 \\
& & & & & & \ddots & \vdots \\
& & & & & & & 0
\end{array}\right]
$$

Then:

(a1.1) If $a_{11}=a_{j j}=0$, it is easy to prove that $r\left(A_{2}^{2}\right)=0$. The Jordan form of the completion $A_{2_{0}}$ consists of $n-2$ blocks of size $q_{1}=q_{2}=2$ and $q_{3}=\cdots=q_{n-2}=1$. Therefore, $r(A)=r\left(A_{2}\right)=\operatorname{rank}\left(A_{2_{0}}\right)=2$ and $r\left(A^{2}\right)=r\left(A_{2}^{2}\right)=\operatorname{rank}\left(A_{2_{0}}^{2}\right)=0$.

If the sequence $\left\{n_{i}\right\}_{i=1}^{p}$ satisfies condition (2), then it majorizes the sequence $\left\{q_{i}\right\}_{i=1}^{n-2}$. By performing the permutation

$$
(1, n, 2,3, \ldots, j-1, j, t, j+1, \ldots, t-1, t+1, \ldots, n-1)
$$

of rows and columns in the matrix $A_{2}$ and by applying the algorithm of [6] to the new matrix (see Appendix, Part I) we obtain a completion of the matrix $A_{2}$ such that its 
Jordan form consists of $p$ blocks of size $n_{i}, i=1,2, \ldots, p$. This completion guarantees the existence of one completion of the matrix $A$ with the desired characteristics.

(a1.2) If $a_{11} \neq 0$, we can transform, by lower similarity, the matrix $A_{2}$ into the following matrix

$$
A_{3}=\left[\begin{array}{cccccccc}
0 & 0 & \cdots & a_{1 j} & \cdots & a_{1 t} & \cdots & a_{1 n} \\
& 0 & \cdots & 0 & \cdots & 0 & \cdots & 0 \\
& & \ddots & \vdots & & \vdots & & \vdots \\
& & & 0 & \cdots & 0 & \cdots & a_{j n} \\
& & & & \ddots & \vdots & & \vdots \\
& & & & & 0 & \cdots & 0 \\
& & & & & & \ddots & \vdots \\
& & & & & & & 0
\end{array}\right] .
$$

This matrix satisfies that $r\left(A_{3}\right)=2$ and $r\left(A_{3}^{2}\right)=1$. The Jordan form of the completion $A_{3_{0}}$ consists of $n-2$ blocks of size $q_{1}=3, q_{2}=\cdots=q_{n-2}=1$. Therefore, $r(A)=r\left(A_{3}\right)=\operatorname{rank}\left(A_{3_{0}}\right)=2, r\left(A^{2}\right)=r\left(A_{3}^{2}\right)=\operatorname{rank}\left(A_{3_{0}}^{2}\right)=1$ and $r\left(A^{3}\right)=r\left(A_{3}^{3}\right)=\operatorname{rank}\left(A_{3_{0}}^{3}\right)=0$.

If the sequence $\left\{n_{i}\right\}_{i=1}^{p}$ satisfies condition (2), then it majorizes the sequence $\left\{q_{i}\right\}_{i=1}^{n-2}$. By applying the method given in Appendix, Part II, we obtain a completion of the matrix $A_{3}$, such that its Segre characteristic is $\left\{n_{i}\right\}_{i=1}^{p}$.

(a2) $a_{j j}$ is the only nonzero entry in the jth row.

By lower similarity we can transform the matrix $A_{1}$ into

$$
A_{2}=\left[\begin{array}{cccccc}
0 & 0 & \cdots & a_{1 j} & \cdots & a_{1 n} \\
& 0 & \cdots & 0 & \cdots & 0 \\
& & \ddots & \vdots & & \vdots \\
& & & 0 & \cdots & a_{j n} \\
& & & & \ddots & \vdots \\
& & & & & 0
\end{array}\right]
$$

It is easily proved that the Jordan form of the completion $A_{2_{0}}$ consists of $n-2$ blocks of size $q_{1}=3$ and $q_{2}=\cdots=q_{n-2}=1$, and that if the sequence $\left\{n_{i}\right\}_{i=1}^{p}$ satisfies condition (2), then it majorizes the sequence $\left\{q_{i}\right\}_{i=1}^{n-2}$. By performing the permutation $(1, j, n, 2,3, \ldots, n-1)$ of rows and columns in the matrix $A_{2}$, and by applying the algorithm of [6] (see Appendix, Part I) we obtain a completion of the matrix $A_{2}$ such that its Segre characteristic is the sequence $\left\{n_{i}\right\}_{i=1}^{p}$.

(b) $a_{1 n}=0$ and $a_{j n} \neq 0$. 
By lower similarity we transform the matrix $A_{1}$ into

$$
A_{2}=\left[\begin{array}{ccccccccc}
a_{11} & 0 & \cdots & a_{1 j} & 0 & \cdots & a_{1 t} & \cdots & 0 \\
& 0 & \cdots & 0 & 0 & \cdots & 0 & \cdots & 0 \\
& & \ddots & \vdots & \vdots & & \vdots & & \vdots \\
& & & a_{j j} & 0 & \cdots & 0 & \cdots & a_{j n} \\
& & & & 0 & \cdots & 0 & \cdots & 0 \\
& & & & & \ddots & \vdots & & \vdots \\
& & & & & & 0 & \cdots & 0 \\
& & & & & & & \ddots & \vdots \\
& & & & & & & 0
\end{array}\right],
$$

where $a_{1 t}$ is the first nonzero entry in the first row, starting from the right. Now, we can distinguish the following subcases.

(b1) $a_{11}=a_{j j}=0$.

If $a_{1 j}=0$ the Jordan form of the completion $A_{2_{0}}$ consists of $n-2$ blocks of size $q_{1}=q_{2}=2$ and $q_{3}=\cdots=q_{n-2}=1$. Again, if the sequence $\left\{n_{i}\right\}_{i=1}^{p}$ satisfies (2), then it majorizes the sequence $\left\{q_{i}\right\}_{i=1}^{n-2}$ and we obtain the desired completion by applying the algorithm of [6] to the matrix obtained performing the permutation

$$
(1, t, 2,3, \ldots, j-1, j, n, j+1, \ldots, t-1, t+1, \ldots, n-1)
$$

of rows and columns in the matrix $A_{2}$ (see Appendix, Part I).

If $a_{1 j} \neq 0$ we apply the method given in (a1.2), to the matrix $A_{2}$.

(b2) $a_{11} \neq 0$ and $a_{j j} \neq 0$.

If $a_{1 j}=0$ we transform, by lower similarity, the matrix $A_{2}$ into

$$
A_{3}=\left[\begin{array}{cccccccc}
0 & 0 & \cdots & a_{1 j} & \cdots & a_{1 t} & \cdots & 0 \\
& 0 & \cdots & 0 & \cdots & 0 & \cdots & 0 \\
& & \ddots & \vdots & & \vdots & & \vdots \\
& & & 0 & \cdots & a_{j t} & \cdots & a_{j n} \\
& & & & \ddots & \vdots & & \vdots \\
& & & & & 0 & \cdots & a_{t n} \\
& & & & & & \ddots & \vdots \\
& & & & & & & 0
\end{array}\right] .
$$

We prove easily that the Jordan form of the completion $A_{3_{0}}$ consists of $n-3$ blocks of size $q_{1}=4$ and $q_{2}=\cdots=q_{n-3}=1$, and again that if the sequence $\left\{n_{i}\right\}_{i=1}^{p}$ satisfies (2), then it majorizes the sequence $\left\{q_{i}\right\}_{i=1}^{n-3}$. By performing the permutation $(1, j, t, n, 2,3, \ldots, n-1)$ of rows and columns in the matrix $A_{3}$, and by applying the algorithm of [6] to this new matrix (see Appendix, Part I) we achieve the desired completion.

If $a_{1 j} \neq 0$, by applying lower similarity to the matrix $A_{3}$ we obtain a new matrix as in the case (b1) with $a_{1 j} \neq 0$. 
The next theorem proves that the above conjecture is true for matrices of size $5 \times 5$.

THEOREM 2.2. Let $A$ be a lower irreducible partial upper triangular matrix of size $5 \times 5$ such that trace $(A)=0$. Let $n_{1} \geq n_{2} \geq \cdots \geq n_{p} \geq 1$ be a set of $p$ positive integers such that $\sum_{i=1}^{p} n_{i}=5$. If

$$
r\left(A^{k}\right) \leq \sum_{i: n_{i} \geq k}\left(n_{i}-k\right), \quad k=1,2, \ldots, n_{1},
$$

then there exists a nilpotent completion $A_{c}$ of $A$ whose Jordan form consists of $p$ blocks of sizes $n_{i} \times n_{i}, i=1,2, \ldots, p$.

Proof. Rodman and Shalom proved this theorem for $r(A)=1$ in [9]. As we have seen in Theorem 2.1, this result is also true when $r(A)=2$. If $r(A)=4$ the result follows by applying Theorem 2.1 of [9]. Therefore, we can assume that $r(A)=3$.

If $r\left(A^{4}\right)>0$ and $r\left(A^{5}\right)=0$, the only possible Jordan form for some nilpotent completion of $A$ is $J_{5}(0)$. The existence of this completion is guaranteed by Rodman and Shalom in [9].

If $r\left(A^{3}\right)>0$ and $r\left(A^{4}\right)=0$, there are two possible Jordan forms for some nilpotent completion of $A, J_{4}(0) \oplus J_{1}(0)$ and $J_{5}(0)$. The last one exists by Theorem 2.1 of [9]. The existence of the first one is assured by the initial conditions about minimal rank for $A^{3}$ and $A^{4}$.

Now, assume that $r\left(A^{2}\right)>0$ and $r\left(A^{3}\right)=0$. There are three possible Jordan forms for some nilpotent completion of $A, J_{3}(0) \oplus J_{2}(0), J_{4}(0) \oplus J_{1}(0)$ and $J_{5}(0)$. Again, the last one exists by Theorem 2.1 of [9]. The first structure exists as well because otherwise the minimal rank of $A^{3}$ cannot be zero. Therefore, we can assure that $r\left(A^{2}\right)=1$. Next we prove the existence of a nilpotent completion of the matrix $A$ such that its Segre characteristic is $\{4,1\}$.

Let us assume that $A_{c_{0}}$ is a completion of $A$ such that its Segre characteristic is $\{3,2\}$. Then, we distinguish the following cases:

(a) If the three first rows of $A_{c_{0}}$ are linear independent, this matrix is lower similar to

$$
A_{c_{0}} \sim\left[\begin{array}{ccc|cc}
a_{11} & a_{12} & a_{13} & a_{14} & a_{15} \\
c_{21} & a_{22} & a_{23} & a_{24} & a_{25} \\
c_{31} & c_{32} & a_{33} & a_{34} & a_{35} \\
\hline 0 & 0 & 0 & 0 & 0 \\
0 & 0 & 0 & 0 & 0
\end{array}\right]=\left[\begin{array}{cc}
A_{1} & A_{2} \\
0 & 0
\end{array}\right] .
$$

Since $\operatorname{rank}\left(A_{c_{0}}^{2}\right)=1$ and $\operatorname{rank}\left(A_{c_{0}}^{3}\right)=0$ we can find the following cases:

(a1) $A_{1} \neq 0, A_{1}^{2} \neq 0$ and $A_{1}^{3}=0$.

In this case there exists a nonsingular matrix $T_{1}$ such that $T_{1} A_{1} T_{1}^{-1}=J_{3}(0)$. Therefore,

$$
\left[\begin{array}{cc}
T_{1} & 0 \\
0 & I
\end{array}\right]\left[\begin{array}{cc}
A_{1} & A_{2} \\
0 & 0
\end{array}\right]\left[\begin{array}{cc}
T_{1}^{-1} & 0 \\
0 & I
\end{array}\right]=\left[\begin{array}{ccc|cc}
0 & 1 & 0 & a_{1} & b_{1} \\
0 & 0 & 1 & a_{2} & b_{2} \\
0 & 0 & 0 & a_{3} & b_{3} \\
\hline 0 & 0 & 0 & 0 & 0 \\
0 & 0 & 0 & 0 & 0
\end{array}\right]
$$


where $a_{3} \neq 0$ or $b_{3} \neq 0$, because $\operatorname{rank}\left(A_{c_{0}}\right)=3$. But from the last expression we can assure that $J_{A_{c_{0}}}=J_{4}(0) \oplus J_{1}(0)$, which is a contradiction.

(a2) $A_{1} \neq 0$ and $A_{1}^{2}=0$.

In this case there exists a nonsingular matrix $T_{1}$ such that $T_{1} A_{1} T_{1}^{-1}=J_{2}(0) \oplus J_{1}(0)$.

Therefore,

$$
\left[\begin{array}{cc}
T_{1} & 0 \\
0 & I
\end{array}\right]\left[\begin{array}{cc}
A_{1} & A_{2} \\
0 & 0
\end{array}\right]\left[\begin{array}{cc}
T_{1}^{-1} & 0 \\
0 & I
\end{array}\right]=\left[\begin{array}{ccc|cc}
0 & 1 & 0 & a_{1} & b_{1} \\
0 & 0 & 0 & a_{2} & b_{2} \\
0 & 0 & 0 & a_{3} & b_{3} \\
\hline 0 & 0 & 0 & 0 & 0 \\
0 & 0 & 0 & 0 & 0
\end{array}\right]
$$

where

$$
\operatorname{rank}\left[\begin{array}{ll}
a_{2} & b_{2} \\
a_{3} & b_{3}
\end{array}\right]=2
$$

Next, consider the partial matrix

$$
\bar{A}=\left[\begin{array}{ccc|cc}
a_{11} & a_{12} & a_{13} & a_{14} & a_{15} \\
c_{21} & a_{22} & a_{23} & a_{24} & a_{25} \\
c_{31} & c_{32} & a_{33} & a_{34} & a_{35} \\
\hline 0 & 0 & 0 & 0 & 0 \\
x_{51} & x_{52} & x_{53} & x_{54} & 0
\end{array}\right]
$$

Then,

$$
\left[\begin{array}{cc}
T_{1} & 0 \\
0 & I
\end{array}\right] \bar{A}\left[\begin{array}{cc}
T_{1}^{-1} & 0 \\
0 & I
\end{array}\right]=\left[\begin{array}{ccc|cc}
0 & 1 & 0 & a_{1} & b_{1} \\
0 & 0 & 0 & a_{2} & b_{2} \\
0 & 0 & 0 & a_{3} & b_{3} \\
\hline 0 & 0 & 0 & 0 & 0 \\
\bar{x}_{51} & \bar{x}_{52} & \bar{x}_{53} & \bar{x}_{54} & 0
\end{array}\right]
$$

with

$$
\operatorname{rank}\left[\begin{array}{cc}
a_{2} & b_{2} \\
a_{3} & b_{3}
\end{array}\right]=2 .
$$

If $b_{2} \neq 0$ we take $\bar{x}_{54}=1$ and $\bar{x}_{51}=\bar{x}_{52}=\bar{x}_{53}=0$. The Jordan form of the matrix obtained is $J_{4}(0) \oplus J_{1}(0)$. This matrix allows us to achieve a completion of the initial matrix $A$.

If $b_{2}=0$ we take $\bar{x}_{52}=1$ and $\bar{x}_{51}=\bar{x}_{53}=\bar{x}_{54}=0$. The Jordan form of the matrix obtained is $J_{4}(0) \oplus J_{1}(0)$. Again, this matrix allows us to get a completion of the initial matrix $A$.

(b) If the independent rows of $A_{c_{0}}$ are the first one, the second one and the fourth one, this matrix is lower similar to

$$
A_{c_{0}} \sim\left[\begin{array}{ccccc}
a_{11} & a_{12} & a_{13} & a_{14} & a_{15} \\
c_{21} & a_{22} & a_{23} & a_{24} & a_{25} \\
0 & 0 & 0 & 0 & 0 \\
c_{41} & c_{42} & c_{43} & a_{44} & a_{45} \\
0 & 0 & 0 & 0 & 0
\end{array}\right]
$$


By performing the permutation $(1,2,4,3,5)$ of rows and columns in $A_{c_{0}}$ and by applying the above method, we obtain the desired completion.

(c) Finally, if the independent rows of $A_{c_{0}}$ are the first one, the third one and the fourth one, this matrix is lower similar to

$$
A_{c_{0}} \sim\left[\begin{array}{ccccc}
a_{11} & a_{12} & a_{13} & a_{14} & a_{15} \\
0 & 0 & 0 & 0 & 0 \\
c_{31} & c_{32} & a_{33} & a_{34} & a_{35} \\
c_{41} & c_{42} & c_{43} & a_{44} & a_{45} \\
0 & 0 & 0 & 0 & 0
\end{array}\right] .
$$

Similarly, by performing the permutation $(1,3,4,2,5)$ of rows and columns in $A_{c_{0}}$ and by applying the method given in $(a)$ we achieve the desired completion. $\square$

3. Appendix. Part I: Let $Y$ be a matrix of size $n \times m$ such that its $m$-diagonal sums are $s_{1}, s_{2}, \ldots s_{m}$. It is well known, see [10], that the matrix

$$
\left[\begin{array}{cc}
J_{m} & O \\
Y & J_{n}
\end{array}\right] \text { is lower similar to }\left[\begin{array}{cc}
J_{m} & O \\
L & J_{n}
\end{array}\right] \text {, }
$$

where

$$
L=\left[\begin{array}{cccc}
0 & 0 & \ldots & 0 \\
0 & 0 & \ldots & 0 \\
\vdots & \vdots & & \vdots \\
0 & 0 & \ldots & 0 \\
s_{1} & s_{2} & \ldots & s_{m}
\end{array}\right]
$$

The matrix $L$ is said to be the lower concentrated form of the matrix $Y$.

In the same way, if $c_{1}, c_{2}, \ldots, c_{n}$ are the $n$-diagonal sums the matrix

$$
\left[\begin{array}{cc}
J_{m} & O \\
Y & J_{n}
\end{array}\right] \text { is lower similar to }\left[\begin{array}{cc}
J_{m} & O \\
C & J_{n}
\end{array}\right] \text {, }
$$

where

$$
C=\left[\begin{array}{cccc}
c_{1} & 0 & \ldots & 0 \\
c_{2} & 0 & \ldots & 0 \\
\vdots & \vdots & & \vdots \\
c_{n} & 0 & \ldots & 0
\end{array}\right]
$$

The matrix $C$ is called left concentrated form of the matrix $Y$.

In general given a matrix

$$
A=\left[\begin{array}{ccccc}
J_{n_{1}} & O & \cdots & O & O \\
Y_{21} & J_{n_{2}} & \cdots & O & O \\
\vdots & \vdots & & \vdots & \vdots \\
Y_{p-11} & Y_{p-12} & \cdots & J_{n_{p-1}} & O \\
Y_{p 1} & Y_{p 2} & \cdots & Y_{p p-1} & J_{n_{p}}
\end{array}\right]
$$


by lower similarity we can transform $A$ into the matrix

$$
\bar{A}=\left[\begin{array}{ccccc}
J_{n_{1}} & O & \cdots & O & O \\
C_{21} & J_{n_{2}} & \cdots & O & O \\
\vdots & \vdots & & \vdots & \vdots \\
C_{p-11} & C_{p-12} & \cdots & J_{n_{p-1}} & O \\
C_{p 1} & C_{p 2} & \cdots & C_{p p-1} & J_{n_{p}}
\end{array}\right],
$$

where

$$
C_{i j}=\left[\begin{array}{cccc}
c_{11}^{(i j)} & 0 & \ldots & 0 \\
c_{21}^{(i j)} & 0 & \ldots & 0 \\
\vdots & \vdots & & \vdots \\
c_{n_{i} 1}^{(i j)} & 0 & \ldots & 0
\end{array}\right]_{n_{i} \times n_{j}}
$$

for $i>j, i=2,3, \ldots, p$ and $j=1,2, \ldots, p-1$, and $C_{i i-1}$ is the left concentrated form of the matrix $Y_{i i-1}$, for $i=2,3, \ldots, p$. We call the matrix $\bar{A}$ block reduction to the first column of the matrix $A$.

The algorithm given in [6] is designed to work on a partial block upper triangular matrices, that is, matrices with the following structure

$$
\tilde{A}=\left[\begin{array}{ccccc}
J_{n_{1}} & O & \cdots & O & O \\
X_{21} & J_{n_{2}} & \cdots & O & O \\
\vdots & \vdots & & \vdots & \vdots \\
X_{p-11} & X_{p-12} & \cdots & J_{n_{p-1}} & O \\
X_{p 1} & X_{p 2} & \cdots & X_{p p-1} & J_{n_{p}}
\end{array}\right],
$$

where the matrix $X_{i j}$ has all its elements unknown, for $i>j, i=2,3, \ldots, p$ and $j=1,2, \ldots, p-1$.

Now consider the following partial block matrix

$$
M_{1}=\left[\begin{array}{ccccc}
D_{1} & E_{12} & \cdots & E_{1 p-1} & E_{1 p} \\
B_{21} & D_{2} & \cdots & E_{2 p-1} & E_{2 p} \\
\vdots & \vdots & & \vdots & \vdots \\
B_{p-11} & B_{p-12} & \cdots & D_{p-1} & E_{p-1 p} \\
B_{p 1} & B_{p 2} & \cdots & B_{p p-1} & D_{p}
\end{array}\right],
$$

where

- For $i=1,2, \ldots, p$

$$
D_{i}=\left[\begin{array}{cccccc}
0 & a_{12}^{(i)} & a_{13}^{(i)} & \cdots & a_{1 n_{i-1}}^{(i)} & a_{1 n_{i}}^{(i)} \\
x & 0 & a_{23}^{(i)} & \cdots & a_{2 n_{i-1}}^{(i)} & a_{2 n_{i}}^{(i)} \\
x & x & 0 & \cdots & a_{3 n_{i-1}}^{(i)} & a_{3 n_{i}}^{(i)} \\
\vdots & \vdots & \vdots & & \vdots & \vdots \\
x & x & x & \cdots & 0 & a_{n_{i-1} n_{i}}^{(i)} \\
x & x & x & \cdots & x & 0
\end{array}\right]_{n_{i} \times n_{i}}
$$


and $\operatorname{rank}\left(D_{i_{0}}\right)=n_{i}-1$.

- For $i>j, i=2,3, \ldots, p$ and $j=1,2, \ldots, p-1$, the matrix $B_{i j}$ has at least its first column formed by unspecified elements, being the rest of its elements unspecified or zero.

- For $i<j, i=1,2, \ldots, p-1$ and $j=2,3, \ldots, p$, the elements of block $E_{i j}$ can be unknown or equal to zero.

It is easy to see that the Segre characteristic of the completion $M_{1_{0}}$ is $\left\{n_{1}, n_{2}, \ldots, n_{p}\right\}$. Let $\left\{m_{j}\right\}_{j=1}^{s}$ be a sequence such that $\left\{n_{i}\right\}_{i=1}^{p} \prec\left\{m_{j}\right\}_{j=1}^{s}$. We obtain a completion of the matrix $M_{1}$ such that its Segre characteristic is $\left\{m_{j}\right\}_{j=1}^{s}$ by applying the following process:

(a) Replace by zeros all the unspecified elements of $M_{1}$, except the elements in the first column of blocks $B_{i j}$, for $i>j, i=2,3, \ldots, p$ and $j=1,2, \ldots, p-1$. Therefore we obtain:

$$
M_{2}=\left[\begin{array}{cccccc}
D_{1_{0}} & O & O & \cdots & O & O \\
\tilde{B}_{21} & D_{2_{0}} & O & \cdots & O & O \\
\tilde{B}_{31} & \tilde{B}_{32} & D_{3_{0}} & \cdots & O & O \\
\vdots & \vdots & & \vdots & \vdots & \vdots \\
\tilde{B}_{p 1} & \tilde{B}_{p 2} & \tilde{B}_{p 3} & \cdots & \tilde{B}_{p p-1} & D_{p_{0}}
\end{array}\right] .
$$

(b) By admissible similarity, the matrix $M_{2}$ is transformed into the matrix

$$
M_{3}=T^{-1} M_{2} T=\left[\begin{array}{ccccc}
J_{n_{1}} & O & O & \cdots & O \\
S_{21} & J_{n_{2}} & O & \cdots & O \\
S_{31} & S_{32} & J_{n_{3}} & \cdots & O \\
\vdots & \vdots & \vdots & & \vdots \\
S_{p 1} & S_{p 2} & S_{p 3} & \cdots & J_{n_{p}}
\end{array}\right]
$$

where the matrix $S_{i j}$, for $i>j, i=2,3, \ldots, p$ and $j=1,2, \ldots, p-1$, has its first column formed by unknown elements, and the other entries equal to zero.

(c) Apply the Algorithm given in [6] to the matrix $\tilde{A}$ in order to obtain a completion $\tilde{A}_{c}$ such that its Segre characteristic is $\left\{m_{j}\right\}_{j=1}^{s}$.

(d) Obtain the block reduction to the first column of $\tilde{A}_{c}$ and call $\tilde{A}_{c_{1}}$ the matrix obtained.

The matrix $\tilde{A}_{c_{1}}$ is also a completion of $M_{3}$. Since the matrix $T$ is an admissible similarity, $T \tilde{A}_{c_{1}} T^{-1}$ is a completion of $M_{2}$ and therefore of $M_{1}$ with the desired characteristic. 
EXAMPLE 3.1. Consider the partial block matrix

$$
M_{1}=\left[\begin{array}{cccc|cc|ccc}
0 & 1 & 1 & 1 & x & 0 & x & x & 0 \\
x & 0 & 2 & 1 & 0 & x & 0 & x & x \\
x & x & 0 & 1 & x & x & x & 0 & x \\
x & x & x & 0 & 0 & x & 0 & x & 0 \\
\hline x & 0 & 0 & 0 & 0 & 2 & x & 0 & x \\
x & x & x & 0 & x & 0 & 0 & x & 0 \\
\hline x & x & 0 & 0 & x & 0 & 0 & 1 & 2 \\
x & x & x & 0 & x & x & x & 0 & 2 \\
x & 0 & x & 0 & x & 0 & x & x & 0
\end{array}\right] .
$$

The Segre characteristic of the matrix $M_{1_{0}}$ is $\{4,2,3\}$. We want to find a completion $M_{1}$ of $M_{1}$ such that its Segre characteristic is the sequence $\{5,4\}$.

Firstly we consider the following matrix

$$
M_{2}=\left[\begin{array}{cccc|cc|ccc}
0 & 1 & 1 & 1 & 0 & 0 & 0 & 0 & 0 \\
0 & 0 & 2 & 1 & 0 & 0 & 0 & 0 & 0 \\
0 & 0 & 0 & 1 & 0 & 0 & 0 & 0 & 0 \\
0 & 0 & 0 & 0 & 0 & 0 & 0 & 0 & 0 \\
\hline x & 0 & 0 & 0 & 0 & 2 & 0 & 0 & 0 \\
x & 0 & 0 & 0 & 0 & 0 & 0 & 0 & 0 \\
\hline x & 0 & 0 & 0 & x & 0 & 0 & 1 & 2 \\
x & 0 & 0 & 0 & x & 0 & 0 & 0 & 2 \\
x & 0 & 0 & 0 & x & 0 & 0 & 0 & 0
\end{array}\right]
$$

By using the admissible similarity

$$
T=\left[\begin{array}{rrrr|rr|rrr}
1 & 0 & 0 & 0 & 0 & 0 & 0 & 0 & 0 \\
0 & 1 & -1 / 2 & 0 & 0 & 0 & 0 & 0 & 0 \\
0 & 0 & 1 / 2 & -1 / 2 & 0 & 0 & 0 & 0 & 0 \\
0 & 0 & 0 & 1 / 2 & 0 & 0 & 0 & 0 & 0 \\
\hline 0 & 0 & 0 & 0 & 1 & 0 & 0 & 0 & 0 \\
0 & 0 & 0 & 0 & 0 & 1 / 2 & 0 & 0 & 0 \\
\hline 0 & 0 & 0 & 0 & 0 & 0 & 1 & 0 & 0 \\
0 & 0 & 0 & 0 & 0 & 0 & 0 & 1 & -1 \\
0 & 0 & 0 & 0 & 0 & 0 & 0 & 0 & 1 / 2
\end{array}\right],
$$

we transform $M_{2}$ into the matrix

$$
M_{3}=T^{-1} M_{2} T=\left[\begin{array}{cccc|cc|ccc}
0 & 1 & 0 & 0 & 0 & 0 & 0 & 0 & 0 \\
0 & 0 & 1 & 0 & 0 & 0 & 0 & 0 & 0 \\
0 & 0 & 0 & 1 & 0 & 0 & 0 & 0 & 0 \\
0 & 0 & 0 & 0 & 0 & 0 & 0 & 0 & 0 \\
\hline x & 0 & 0 & 0 & 0 & 1 & 0 & 0 & 0 \\
x & 0 & 0 & 0 & 0 & 0 & 0 & 0 & 0 \\
\hline x & 0 & 0 & 0 & x & 0 & 0 & 1 & 0 \\
x & 0 & 0 & 0 & x & 0 & 0 & 0 & 1 \\
x & 0 & 0 & 0 & x & 0 & 0 & 0 & 0
\end{array}\right] .
$$


By applying the Algorithm given in [6] to

$$
\tilde{A}=\left[\begin{array}{ccc}
J_{4} & O & O \\
X_{21} & J_{2} & O \\
X_{31} & X_{32} & J_{3}
\end{array}\right]
$$

where $X_{21}, X_{31}$ and $X_{32}$ have all their elements unspecified, we obtain $\tilde{A}_{c}$, which Segre characteristic is $\{5,4\}$. By doing its block reduction to the first column we obtain

$$
\tilde{A}_{c_{1}}=\left[\begin{array}{rrrr|rr|rrr}
0 & 1 & 0 & 0 & 0 & 0 & 0 & 0 & 0 \\
0 & 0 & 1 & 0 & 0 & 0 & 0 & 0 & 0 \\
0 & 0 & 0 & 1 & 0 & 0 & 0 & 0 & 0 \\
0 & 0 & 0 & 0 & 0 & 0 & 0 & 0 & 0 \\
\hline 1 & 0 & 0 & 0 & 0 & 1 & 0 & 0 & 0 \\
0 & 0 & 0 & 0 & 0 & 0 & 0 & 0 & 0 \\
\hline 0 & 0 & 0 & 0 & 0 & 0 & 0 & 1 & 0 \\
0 & 0 & 0 & 0 & 1 & 0 & 0 & 0 & 1 \\
-1 & 0 & 0 & 0 & 0 & 0 & 0 & 0 & 0
\end{array}\right] .
$$

The matrix

$$
M_{1_{c}}=T \tilde{A}_{c_{1}} T^{-1}=\left[\begin{array}{cccc|cc|ccc}
0 & 1 & 1 & 1 & 0 & 0 & 0 & 0 & 0 \\
0 & 0 & 2 & 1 & 0 & 0 & 0 & 0 & 0 \\
0 & 0 & 0 & 1 & 0 & 0 & 0 & 0 & 0 \\
0 & 0 & 0 & 0 & 0 & 0 & 0 & 0 & 0 \\
\hline 1 & 0 & 0 & 0 & 0 & 2 & 0 & 0 & 0 \\
0 & 0 & 0 & 0 & 0 & 0 & 0 & 0 & 0 \\
\hline 0 & 0 & 0 & 0 & 0 & 0 & 0 & 1 & 2 \\
1 & 0 & 0 & 0 & 1 & 0 & 0 & 0 & 2 \\
-1 / 2 & 0 & 0 & 0 & 0 & 0 & 0 & 0 & 0
\end{array}\right]
$$

is a completion of $M_{1}$ with the desired characteristic.

Part II: Consider the following lower irreducible partial upper triangular matrix,

$$
A=\left[\begin{array}{cccccccc}
0 & 0 & \cdots & a_{1 j} & \cdots & a_{1 t} & \cdots & a_{1 n} \\
& 0 & \cdots & 0 & \cdots & 0 & \cdots & 0 \\
& & \ddots & \vdots & & \vdots & & \vdots \\
& & & 0 & \cdots & 0 & \cdots & a_{j n} \\
& & & & \ddots & \vdots & & \vdots \\
& & & & & 0 & \cdots & 0 \\
& & & & & & \ddots & \vdots \\
& & & & & & & 0
\end{array}\right] .
$$

This matrix satisfies that $r(A)=2, r\left(A^{2}\right)=1$ and $r\left(A^{3}\right)=0$. The Jordan form of the completion $A_{0}$ consists of $n-2$ blocks of size $q_{1}=3$ and $q_{i}=1, i=2,3, \ldots, n-2$. Therefore, $r(A)=\operatorname{rank}\left(A_{0}\right)=2, r\left(A^{2}\right)=\operatorname{rank}\left(A_{0}^{2}\right)=1$ and $r\left(A^{3}\right)=\operatorname{rank}\left(A_{0}^{3}\right)=0$. 
If the sequence $\left\{n_{i}\right\}_{i=1}^{p}$ satisfies condition (2) then it majorizes the sequence $\left\{q_{i}\right\}_{i=1}^{n-2}$. We are going to obtain a completion $A_{c}$ of the matrix $A$ whose Segre characteristic is $\left\{n_{i}\right\}_{i=1}^{p}$.

By performing the permutation $(1, j, n, 2, \ldots, j-1, j+1, \ldots, n-1)$ of rows and columns in $A$, we obtain

$$
A_{1}=\left[\begin{array}{ll}
A_{11} & A_{12} \\
A_{21} & A_{22}
\end{array}\right]
$$

where

$$
A_{11}=\left[\begin{array}{ccc}
0 & a_{1 j} & a_{1 n} \\
* & 0 & a_{j n} \\
* & * & 0
\end{array}\right], A_{12}=\left[\begin{array}{cccccccc}
0 & \cdots & 0 & 0 & \cdots & a_{1 t} & \cdots & 0 \\
* & \cdots & * & 0 & \cdots & 0 & \cdots & 0 \\
* & \cdots & * & * & \cdots & * & \cdots & *
\end{array}\right],
$$

$A_{21}$ is a matrix of size $(n-3) \times 3$ whose first column is formed by unspecified elements and $A_{22}$ is a partial upper triangular matrix whose known elements are equal to zero. Next, consider the matrix

$$
\bar{A}_{1}=\left[\begin{array}{ll}
A_{11} & \bar{A}_{12} \\
A_{21} & A_{22}
\end{array}\right]
$$

where $\bar{A}_{12}$ is like $A_{12}$ with $a_{1 t}=0$. The structure of $\bar{A}_{1}$ allows us to apply the algorithm given in [6] and to obtain a completion

$$
\bar{A}_{1_{c}}=\left[\begin{array}{cc}
A_{11_{0}} & 0 \\
A_{21_{c}} & A_{22_{c}}
\end{array}\right]
$$

whose Segre characteristic is $\left\{n_{i}\right\}_{i=1}^{p}$. We can distinguish two cases:

(a) The row $t+1$ is zero.

Then the matrix

$$
A_{1_{c}}=\left[\begin{array}{ll}
A_{11_{0}} & A_{12_{0}} \\
A_{21_{c}} & A_{22_{c}}
\end{array}\right]
$$

is a completion of $A_{1}$ similar to $\bar{A}_{1_{c}}$.

(b) The row $t+1$ of $\bar{A}_{1_{c}}$ has an 1 .

In this case, consider the matrix

$$
A_{2}=\left[\begin{array}{cc}
\tilde{A}_{11} & A_{12} \\
A_{21_{c}} & A_{22_{c}}
\end{array}\right],
$$

where $\tilde{A}_{11}$ is like $A_{11}$ but its entry $(3,2)$ is equal to 0 . We are going to find a completion $A_{2 c}$ similar to $\bar{A}_{1_{c}}$. If the 1 of $\bar{A}_{1_{c}}$ is in position $(t+1,1)$, the matrix $A_{2_{c}}$ is

$$
A_{2 c}=\left[\begin{array}{ll}
\tilde{A}_{11_{c}} & A_{12_{0}} \\
A_{21_{c}} & A_{22_{c}}
\end{array}\right]
$$


where

$$
\tilde{A}_{11_{c}}=\left[\begin{array}{ccc}
0 & a_{1 j} & a_{1 n} \\
\alpha & 0 & a_{j n} \\
0 & 0 & 0
\end{array}\right], \quad \text { with } \alpha=-a_{1 t} / a_{1 j} \text {. }
$$

If the 1 of $\bar{A}_{1_{c}}$ is in position $(t+1, h)$, with $3<h \leq j+1$, the matrix $A_{2_{c}}$ is

$$
A_{2_{c}}=\left[\begin{array}{ll}
\tilde{A}_{11_{0}} & A_{12_{c}} \\
A_{21_{c}} & A_{22_{c}}
\end{array}\right]
$$

where

$$
A_{12_{c}}=\left[\begin{array}{cccccccc}
0 & \cdots & 0 & 0 & \cdots & a_{1 t} & \cdots & 0 \\
0 & \cdots & \alpha & 0 & \cdots & 0 & \cdots & 0 \\
0 & \cdots & 0 & 0 & \cdots & 0 & \cdots & 0
\end{array}\right]
$$

with $\alpha=-a_{1 t} / a_{1 j}$ in position $(2, h)$.

Finally, if the 1 is in position $(t+1, h)$, with $j+1<h \leq t$, by elementary transformations we obtain a completion $A_{2 c}$ similar to $\bar{A}_{1_{c}}$.

EXample 3.2. Consider the partial upper triangular matrix

$$
A=\left[\begin{array}{llllllll}
0 & 0 & 1 & 0 & 0 & 1 & 0 & 1 \\
* & 0 & 0 & 0 & 0 & 0 & 0 & 0 \\
* & * & 0 & 0 & 0 & 0 & 0 & 1 \\
* & * & * & 0 & 0 & 0 & 0 & 0 \\
* & * & * & * & 0 & 0 & 0 & 0 \\
* & * & * & * & * & 0 & 0 & 0 \\
* & * & * & * & * & * & 0 & 0 \\
* & * & * & * & * & * & * & 0
\end{array}\right]
$$

We are going to find a completion of $A$ such that its Segre characteristic is $\{7,1\}$. First, by performing the permutation $(1,3,8,2,4,5,6,7)$ we obtain the matrix

$$
A_{1}=\left[\begin{array}{lll|lllll}
0 & 1 & 1 & 0 & 0 & 0 & 1 & 0 \\
* & 0 & 1 & * & 0 & 0 & 0 & 0 \\
* & * & 0 & * & * & * & * & * \\
\hline * & 0 & 0 & 0 & 0 & 0 & 0 & 0 \\
* & * & 0 & * & 0 & 0 & 0 & 0 \\
* & * & 0 & * & * & 0 & 0 & 0 \\
* & * & 0 & * & * & * & 0 & 0 \\
* & * & 0 & * & * & * & * & 0
\end{array}\right] .
$$

By applying the algorithm of [6] to the matrix $\bar{A}_{1}$ we obtain the following matrix 
whose Segre characteristic is $\{7,1\}$

$$
\bar{A}_{1_{c}}=\left[\begin{array}{ccc|ccccc}
0 & 1 & 1 & 0 & 0 & 0 & 0 & 0 \\
0 & 0 & 1 & 0 & 0 & 0 & 0 & 0 \\
0 & 0 & 0 & 0 & 0 & 0 & 0 & 0 \\
\hline 1 & 0 & 0 & 0 & 0 & 0 & 0 & 0 \\
0 & 0 & 0 & 1 & 0 & 0 & 0 & 0 \\
0 & 0 & 0 & 0 & 1 & 0 & 0 & 0 \\
0 & 0 & 0 & 0 & 0 & 1 & 0 & 0 \\
0 & 0 & 0 & 0 & 0 & 0 & 0 & 0
\end{array}\right] .
$$

Since there is an 1 in the row 7 , consider the matrix

$$
A_{2}=\left[\begin{array}{ccc|ccccc}
0 & 1 & 1 & 0 & 0 & 0 & 1 & 0 \\
* & 0 & 1 & * & 0 & 0 & 0 & 0 \\
* & 0 & 0 & * & * & * & * & * \\
\hline 1 & 0 & 0 & 0 & 0 & 0 & 0 & 0 \\
0 & 0 & 0 & 1 & 0 & 0 & 0 & 0 \\
0 & 0 & 0 & 0 & 1 & 0 & 0 & 0 \\
0 & 0 & 0 & 0 & 0 & 1 & 0 & 0 \\
0 & 0 & 0 & 0 & 0 & 0 & 0 & 0
\end{array}\right] .
$$

By applying the elementary transformations $C 7-C 2 \rightarrow C 7, C 6-C 3+C 2 \rightarrow C 6$ and $C 5+C 3-C 2 \rightarrow C 5$ (where $C i$ is the column $i$ of $A_{2}$ ), and by its corresponding transformations by rows, we obtain the matrix

$$
A_{2_{c}}=\left[\begin{array}{rrr|rrrrr}
0 & 1 & 1 & 0 & 0 & 0 & 1 & 0 \\
0 & 0 & 1 & -1 & 0 & 0 & 0 & 0 \\
0 & 0 & 0 & 1 & -1 & 0 & 0 & 0 \\
\hline 1 & 0 & 0 & 0 & 0 & 0 & 0 & 0 \\
0 & 0 & 0 & 1 & 0 & 0 & 0 & 0 \\
0 & 0 & 0 & 0 & 1 & 0 & 0 & 0 \\
0 & 0 & 0 & 0 & 0 & 1 & 0 & 0 \\
0 & 0 & 0 & 0 & 0 & 0 & 0 & 0
\end{array}\right] .
$$

This matrix is a completion of $A_{2}$, similar to $\bar{A}_{1_{c}}$. By performing the permutation $(1,4,2,5,6,7,8,3)$ of rows and columns in the matrix $A_{2_{c}}$ we obtain a completion $A_{c}$ of the matrix $A$ whose Segre characteristic is $\{7,1\}$.

\section{REFERENCES}

[1] Joseph A. Ball, Israel Gohberg, Leiba Rodman and Tamir Shalom. On the eigenvalues of matrices with given upper triangular part. Integral Equations and Operator Theory, 13:488-497, 1990.

[2] Israel Gohberg and Sorin Rubinstein. A classification of upper equivalent matrices: the generic case. Integral Equations and Operator Theory, 14:533-544, 1991.

[3] Leonid Gurvits, Leiba Rodman and Tamir Shalom. Controllability and completion of partial upper triangular matrices over rings. Linear Algebra and its Applications, 172:135-149, 1992. 
[4] Leonid Gurvits, Leiba Rodman and Tamir Shalom. Controllability of completion of partial upper triangular matrices. Mathematics of Control, Signals and Systems, 6:30-40, 1993.

[5] Cristina Jordán, Juan R. Torregrosa and Ana M. Urbano. On the Jordan Form of Completions of Partial Upper Triangular Matrices. Linear Algebra and its Applications, 254:241-250, 1997.

[6] Cristina Jordán, Juan R. Torregrosa and Ana M. Urbano. An Algorithm for Nilpotent Completions of Partial Jordan Matrices. Linear Algebra and its Applications, 275/276:315-325, 1998.

[7] Mark Krupnik. Geometric multiplicities of completions of partial triangular matrices. Linear Algebra and its Applications, 220:215-227, 1995.

[8] Mark Krupnik and Leiba Rodman. Completions of partial Jordan and Hessenberg matrices. Linear Algebra and its Applications, 212/213:267-287, 1994.

[9] Leiba Rodman and Tamir Shalom. Jordan form of completions of partial upper triangular matrices. Linear Algebra and its Applications, 168:221-249, 1992.

[10] Ion Zaballa. Matrices with prescribed rows and invariant factors. Linear Algebra and its Applications, 87:113-146, 1987. 